Universidad Católica del Norte

Antofagasta - Chile

\title{
On ideal convergence of triple sequences in intuitionistic Fuzzy normed space defined by compact operator
}

\author{
Vakeel A. Khan \\ Aligarh Muslim University, India \\ Mohd. Imran Idrisi \\ Aligarh Muslim University, India \\ and \\ Umme Tuba \\ Aligarh Muslim University, India \\ Received : June 2019. Accepted : April 2021
}

\begin{abstract}
The main purpose of this article is to introduce and study some new spaces of I-convergence of triple sequences in intuitionistic fuzzy normed space defined by compact operator i.e ${ }_{3} \mathcal{S}_{(\mu, \nu)}^{I}(T)$ and ${ }_{3} \mathcal{S}_{0(\mu, \nu)}^{I}(T)$ and examine some fundamental properties, fuzzy topology and verify inclusion relations lying under these spaces.
\end{abstract}

Key words: Ideal, Filter, T-norm, T-conorm, Intuitionistic fuzzy normed spaces. 


\section{Introduction}

In 1986, Atanassov K.T.[13] introduced the idea of Intuitionistic fuzzy set theory which is a generalization of fuzzy set. Fuzzy set theory is a powerful tool for modelling uncertainty and vagueness by imputing the degree of membership to the elements so that individuals can be distinguished in a given set. Immense number of research papers recently surfaced in scientific discipline showing that the fuzzy set theory strangely has turned into the todays norm for young scientists or researchers. Many authors have made the concept of fuzzy topology as a very significant tool towards their work. The idea of intuitionistic fuzzy normed space[15] and intuitionistic fuzzy 2-normed space[20] are the most recent evolutions in fuzzy topology.

In the beginning, the idea of statistical convergence was presented independently by Fast[11] and Schoenberg[12]. I-convergence is a generality of statistical convergence which was introduced by Salat et al. [18]. Later on the idea of statistical convergence for double sequences have been defined by Edely and Mursaleen[16] and Tripathy [6] independently and for fuzzy numbers by Mursaleen and Savaş[10]. Related to this, there are infact two quite different types of convergence i.e $I$ and $I^{*}$-convergence for double sequences [17].

In 2007 Gurdal, Sahiner and Duden[3] introduced the concept of convergence of triple sequences. This concept has been further investigated by many authors, see $([1],[2],[4],[5])$. Tripathy and Goswami used the idea of $I$-convergence of triple sequences in probabilistic normed spaces. Tripathy and Shiner[4] studied the properties associated with $I$-convergence in triple sequence spaces and showed some useful results.

\section{Preliminaries and definitions}

Here, we recall some basic definitions and examples associated to this article.

Definition 1.1:[3] A triple sequence $x=\left(x_{n k l}\right)$ is said to be convergent to a number $\xi$ in pringsheim's sense if for every $\varepsilon_{0}>0, \exists m_{\left(\varepsilon_{0}\right)} \in \mathbf{N}$ such that

$$
\left|x_{n k l}-\xi\right|<\varepsilon_{0} \text { whenever } n \geq m_{\left(\varepsilon_{0}\right)}, \quad k \geq m_{\left(\varepsilon_{0}\right)}, \quad l \geq m_{\left(\varepsilon_{0}\right)} .
$$


Example:[3] Let

$$
x_{n k l}= \begin{cases}k l, & n=3 \\ n l, & k=5, \\ n k, & l=7, \\ 8, & \text { otherwise. }\end{cases}
$$

Then $\left(x_{n k l}\right) \rightarrow 8$ in Pringsheim's sense.

Definition 1.2:[3] A triple sequence $x=\left(x_{n k l}\right)$ is said to be statistically convergent to a number $\xi$ if for each $\varepsilon_{0}>0$,

$$
\delta_{3}\left(\left\{(n, k, l) \in \mathbf{N} \times \mathbf{N} \times \mathbf{N}:\left|x_{n k l}-\xi\right| \geq \varepsilon_{0}\right\}\right)=0
$$

and to be symbolized as, st $\lim _{n, k, l \rightarrow \infty}=\xi$.

Definition 1.3:[22] A family of sets $I \subseteq 2^{X}$ is said to be an ideal for a non-empty set $X$, if it satisfies:

(i) $\phi \in I$;

(ii) if $A, B \in I \Rightarrow A \cup B \in I$;

(iii) if $A \in I$ and $B \subseteq A \Rightarrow B \in I$.

An ideal $I$ is called a non-trivial ideal if $X \notin I$.

Definition 1.4:[22] A family of sets $\mathcal{F} \subseteq 2^{X}$ is said to be a filter for a non-empty set $X$, if it satisfies:

(i) $\phi \notin \mathcal{F}$;

(ii) if $A, B \in \mathcal{F} \Rightarrow A \cap B \in \mathcal{F}$;

(iii) if $A \in \mathcal{F}$ and $A \subseteq B \Rightarrow B \in \mathcal{F}$.

For each ideal $I$ there is a filter $\mathcal{F}(I)$ corresponding to $I$.

$$
\mathcal{F}(I)=\left\{A \subseteq N: A^{c} \in I\right\} \text {, where } A^{c}=N-A .
$$

Definition 1.5:[5] A triple sequence $x=\left(x_{n k l}\right)$ is said to be $I$ convergent to a number $\xi$ if for every $\varepsilon_{0}>0$, such that

$$
\left\{(n, k, l) \in \mathbf{N} \times \mathbf{N} \times \mathbf{N}:\left|x_{n k l}-\xi\right| \geq \varepsilon_{0}\right\} \in I
$$


and to be symbolized as, $I \lim x_{n k l}=\xi$.

Definition 1.6:[23] A triangular norm $(t-$ norm $)$ is defined as a binary operation

$$
*:[0,1] \times[0,1] \longrightarrow[0,1]
$$

if $*$ satisfies :

(i) $*$ is commutative and associative,

(ii) $*$ is continuous,

(iii) $\alpha * 1=\alpha \forall \alpha \in[0,1]$,

(iv) $\alpha_{1} * \beta_{1} \leq \alpha_{2} * \beta_{2}$ whenever $\alpha_{1} \leq \alpha_{2}$ and $\beta_{1} \leq \beta_{2} \forall \alpha_{i}, \beta_{i} \in[0,1]$ $(i=1,2)$.

Examples: (i) $\alpha * \beta=\alpha \beta$ (ii) $\alpha * \beta=\min \{\alpha, \beta\}$.

Definition 1.7: [23] A triangular conorm $(t-$ conorm $)$ is defined as a binary operation

$$
\diamond:[0,1] \times[0,1] \longrightarrow[0,1]
$$

if $\diamond$ satisfies:

(i) $\diamond$ is commutative and associative,

(ii) $\diamond$ is continuous,

(iii) $\alpha \diamond 0=\alpha \forall \alpha \in[0,1]$,

(iii) $\alpha_{1} \diamond \beta_{1} \leq \alpha_{2} \diamond \beta_{2}$ whenever $\alpha_{1} \leq \alpha_{2}$ and $\beta_{1} \leq \beta_{2} \forall \alpha_{i}, \beta_{i} \in[0,1]$ $(i=1,2)$.

Examples: (i) $\alpha \diamond \beta=\min \{\alpha+\beta, 1\}$ (ii) $\alpha \diamond \beta=\max \{\alpha, \beta\}$.

Definition 1.8[23] Functions $\mu, \nu: X \times(0, \infty) \rightarrow[0,1]$ are said to be fuzzy norms on a linear space $X$ over a field $\mathbf{R}(\mathbf{C})$, if for every $x_{1}, x_{2} \in X$ and $t_{1}, t_{2}>0$, they satisfy: 
(a) $\mu\left(x_{1}, t_{1}\right)+\nu\left(x_{1}, t_{1}\right) \leq 1$,

(b) $\mu\left(x_{1}, t_{1}\right)>0$,

(c) $\mu\left(x_{1}, t_{1}\right)=1 \Longleftrightarrow x_{1}=0$,

(d) $\mu\left(\alpha x_{1}, t_{1}\right)=\mu\left(x_{1}, \frac{t_{1}}{|\alpha|}\right) \forall \alpha \neq 0$,

(e) $\mu\left(x_{1}, t_{1}\right) * \mu\left(x_{2}, t_{2}\right) \leq \mu\left(x_{1}+x_{2}, t_{1}+t_{2}\right)$,

(f) $\mu\left(x_{1},.\right):(0, \infty) \rightarrow[0,1]$ is continuous,

(g) $\lim _{t_{1} \rightarrow \infty} \mu\left(x_{1}, t_{1}\right)=1$ and $\lim _{t_{1} \rightarrow 0} \mu\left(x_{1}, t_{1}\right)=0$,

(h) $\nu\left(x_{1}, t_{1}\right)<1$,

(i) $\nu\left(x_{1}, t_{1}\right)=0 \Longleftrightarrow x_{1}=0$,

(j) $\nu\left(\alpha x_{1}, t_{1}\right)=\nu\left(x_{1}, \frac{t_{1}}{|\alpha|}\right) \forall \alpha \neq 0$,

(k) $\nu\left(x_{1}, t_{1}\right) \diamond \nu\left(x_{2}, t_{2}\right) \geq \nu\left(x_{1}+x_{2}, t_{1}+t_{2}\right)$,

(l) $\nu\left(x_{1},.\right):(0, \infty) \rightarrow[0,1]$ is continuous,

(m) $\lim _{t_{1} \rightarrow \infty} \nu\left(x_{1}, t_{1}\right)=0$ and $\lim _{t_{1} \rightarrow 0} \nu\left(x_{1}, t_{1}\right)=1$.

Then the 5-tuple $(X, \mu, \nu, *, \diamond)$ entirely is said to be an intuitionistic fuzzy normed space (abbreviated as IFNS).

Definition 1.9[23] Let $(X, \mu, \nu, *, \diamond)$ be an intuitionistic fuzzy normed space. Then a sequence $x=\left(x_{k}\right)$ is said to be convergent to a number $\xi$ with respect to the intuitionistic norm $(\mu, \nu)$ if for every $\varepsilon_{0}>0$ and $t_{1}>0, \exists k_{o} \in \mathbf{N}$ such that $\mu\left(x_{k}-\xi, t_{1}\right)>1-\varepsilon_{0}$ and $\nu\left(x_{k}-\xi, t_{1}\right)<\varepsilon_{0} \forall$ $k \geq k_{o}$ and to be symbolized as, $(\mu, \nu) \lim x=\xi$.

Definition 1.10:[23] Let $(x, \mu, \nu, *, \diamond)$ be an intuitionistic fuzzy normed space. Then a sequence $x=\left(x_{k}\right)$ is said to be statistically convergent to a number $\xi$ with respect to the intuitionistic norm $(\mu, \nu)$ if for every $\varepsilon_{0}>0$ and $t_{1}>0$, we have

$$
\delta\left(\left\{k \in \mathbf{N}: \mu\left(x_{k}-\xi, t_{1}\right) \leq 1-\varepsilon_{0} \text { or } \nu\left(x_{k}-\xi, t_{1}\right) \geq \varepsilon_{0}\right\}\right)=0
$$


and to be symbolized as, $s t_{(\mu, \nu)}-\lim x_{k}=\xi$.

Definition 1.11:[23] Let $(X, \mu, \nu, *, \diamond)$ be an intuitionistic fuzzy normed space. Then a sequence $x=\left(x_{k}\right)$ is said to be $I$-convergent to a number $\xi$ with respect to the intuitionistic norm $(\mu, \nu)$ if for every $\varepsilon_{0}>0$ and $t_{1}>0$, we have

$$
\left\{k \in \mathbf{N}: \mu\left(x_{k}-\xi, t_{1}\right) \leq 1-\varepsilon_{0} \text { or } \nu\left(x_{k}-\xi, t_{1}\right) \geq \varepsilon_{0}\right\} \in I .
$$

and to be symbolized as, $I_{(\mu, \nu)} \lim x_{k}=\xi$.

The idea of statistical convergence and statistical Cauchy for double sequences in intuitionistic fuzzy normed spaces have been studied by Mursaleen and Mohiuddine. Recently, Khan and Yasmeen[25] introduced intuitionistic Zweier I-convergent double sequence spaces defined by modulus function:

$$
\begin{gathered}
{ }_{2} \mathcal{Z}_{(\mu, \nu)}^{I}(f)=\left\{\left(x_{n k}\right) \in{ }_{2} \omega:\left\{(n, k) \in \mathbf{N} \times \mathbf{N}: f\left(\mu\left(x_{n k}^{\prime \prime}-\xi, t_{1}\right)\right) \leq 1-\varepsilon_{0}\right.\right. \\
\text { or } \left.\left.f\left(\nu\left(x_{n k}^{\prime \prime}-\xi, t_{1}\right)\right) \geq \varepsilon_{0}\right\} \in I_{2}\right\} ; \\
{ }_{2} \mathcal{Z}_{0(\mu, \nu)}^{I}(f)=\left\{\left(x_{n k}\right) \in{ }_{2} \omega:\left\{(n, k) \in \mathbf{N} \times \mathbf{N}: f\left(\mu\left(x_{n k}^{\prime \prime}, t_{1}\right)\right) \leq 1-\varepsilon_{0}\right.\right. \\
\text { or } \left.\left.f\left(\nu\left(x_{n k}^{\prime \prime}, t_{1}\right)\right) \geq \varepsilon_{0}\right\} \in I_{2}\right\} .
\end{gathered}
$$

\section{$I_{3}$-convergence of triple sequences in IFNS}

Definition 2.1: Let $(X, \mu, \nu, *, \diamond)$ be an intuitionistic fuzzy normed space. Then a triple sequence $x=\left(x_{n k l}\right)$ is said to be statistically convergent to a number $\xi$ with respect to the intuitionistic norm $(\mu, \nu)$ if for every $\epsilon_{o}>0$ and $t_{1}>0$, we have

$\delta\left(\left\{(n, k, l) \in \mathbf{N} \times \mathbf{N} \times \mathbf{N}: \mu\left(x_{n k l}-\xi, t_{1}\right) \leq 1-\varepsilon_{0}\right.\right.$ or $\left.\left.\nu\left(x_{n k l}-\xi, t_{1}\right) \geq \varepsilon_{0}\right\}\right)=0$

or equivalently,

$\lim _{p q r} \frac{1}{p q r} \mid\left\{n \leq p, k \leq q, l \leq r: \mu\left(x_{n k l}-\xi, t_{1}\right) \leq 1-\varepsilon_{0}\right.$ or $\left.\nu\left(x_{n k l}-\xi, t_{1}\right) \geq \varepsilon_{0}\right\} \mid=0$

and to be symbolized as, $s t_{(\mu, \nu)}^{3} \lim x_{n k l}=\xi$. 
Definition 2.2: Let $(X, \mu, \nu, *, \diamond)$ be an intuitionistic fuzzy normed space. Then a triple sequence $x=\left(x_{n k l}\right)$ is said to be statistically Cauchy with respect to the intuitionistic norm $(\mu, \nu)$ if for each $\varepsilon_{0}>0$ and $t_{1}>0 \exists$ $p=p_{\left(\varepsilon_{0}\right)}, q=q_{\left(\varepsilon_{0}\right)}, r=r_{\left(\varepsilon_{0}\right)}$ such that, we have

$\delta\left(\left\{(n, k, l) \in \mathbf{N} \times \mathbf{N} \times \mathbf{N}: \mu\left(x_{n k l}-x_{p q r}, t_{1}\right) \leq 1-\varepsilon_{0}\right.\right.$ or $\left.\left.\nu\left(x_{n k l}-x_{p q r}, t_{1}\right) \geq \varepsilon_{0}\right\}\right)=0$.

Definition 2.3: Let $(X, \mu, \nu, *, \diamond)$ be an intuitionistic fuzzy normed space. Then a triple sequence $x=\left(x_{n k l}\right)$ is said to be $I_{3}$-convergent to a number $\xi$ with respect to the intuitionistic norm $(\mu, \nu)$ if for every $\varepsilon_{0}>0$ and $t_{1}>0$, we have

$\left\{(n, k, l) \in \mathbf{N} \times \mathbf{N} \times \mathbf{N}: \mu\left(x_{n k l}-\xi, t_{1}\right) \leq 1-\varepsilon_{0}\right.$ or $\left.\nu\left(x_{n k l}-\xi, t_{1}\right) \geq\right\} \in I_{3}$.

and to be symbolized as, $I_{3}^{(\mu, \nu)} \lim x_{k}=\xi$.

where, $I_{3}$ is a non trivial ideal of $\mathbf{N} \times \mathbf{N} \times \mathbf{N}$.

A Compact Linear Operator is a function $T: V \rightarrow W$ which satisfies two properties:

(i) $T$ is linear;

(ii) $T\left(x_{k}\right)$ has a convergent subsequence in $W$, for every bounded sequence $\left(x_{k}\right) \in V$.

where $V$ and $W$ are normed linear spaces. The set of all bounded linear operators $\mathcal{B}(V, W)$ is normed linear space normed by

$$
\|T\|=\sup _{x \in V,\|x\|=1}\|T x\|
$$

Remark: The set of all compact linear operators $\mathcal{C}(V, W)$ is a closed subspace of $\mathcal{B}(V, W)$ and if $W$ is a Banach space then $\mathcal{C}(V, W)$ is also a Banach space.

In this article, we study on $I$-convergence of triple sequences defined by compact operator in IFNS. We also define an open ball centered at triple sequence with a non-zero radius and study the topology on the defined spaces. 


\section{Main results}

In this section we introduce the following classes of sequence spaces:

$$
\begin{gathered}
{ }_{3} \mathcal{S}_{(\mu, \nu)}^{I}(T)=\left\{\left(x_{n k l}\right) \in{ }_{3} \omega:\left\{(n, k, l) \in \mathbf{N} \times \mathbf{N} \times \mathbf{N}: \mu\left(T\left(x_{n k l}\right)-\xi, t_{1}\right) \leq 1-\varepsilon_{0}\right.\right. \\
\text { or } \left.\left.\nu\left(T\left(x_{n k l}\right)-\xi, t_{1}\right) \geq \varepsilon_{0}\right\} \in I_{3}\right\} ; \\
{ }_{3} \mathcal{S}_{0(\mu, \nu)}^{I}(T)=\left\{\left(x_{n k l}\right) \in{ }_{3} \omega:\left\{(n, k, l) \in \mathbf{N} \times \mathbf{N} \times \mathbf{N}: \mu\left(T\left(x_{n k l}\right), t_{1}\right) \leq 1-\varepsilon_{0}\right.\right. \\
\left.\left.\quad \text { or } \nu\left(T\left(x_{n k l}\right), t_{1}\right) \geq \varepsilon_{0}\right\} \in I_{3}\right\} .
\end{gathered}
$$

We also define;

$$
\begin{gathered}
{ }_{3} \mathcal{B}_{x}\left(\delta, t_{1}\right)(T)=\left\{\left(y_{n k l}\right) \in{ }_{3} \omega:\left\{(n, k, l): \mathbf{N} \times \mathbf{N} \times \mathbf{N}: \mu\left(T(x)-T(y), t_{1}\right)>1-\delta\right.\right. \\
\text { and } \left.\left.\nu\left(T(x)-T(y), t_{1}\right)<\delta\right\} \in I_{3}\right\}
\end{gathered}
$$

which is an open ball with centre at $x=\left(x_{n k l}\right)$ and radius $\delta$ with respect to $t_{1}$.

Theorem 3.1: If a triple sequence $x=\left(x_{n k l}\right) \in{ }_{3} \mathcal{S}_{(\mu, \nu)}^{I}(T)$ is $I_{3}$-convergent to a number $\xi$ with respect to the intuitionistic norm $(\mu, \nu)$, then the limit $\xi$ is unique.

Proof: Let $x=\left(x_{n k l}\right) \in{ }_{3} \mathcal{S}_{(\mu, \nu)}^{I}(T)$ such that

$$
I_{3}^{(\mu, \nu)} \lim x_{n k l}=\xi_{1} \text { and } I_{3}^{(\mu, \nu)} \lim x_{n k l}=\xi_{2} .
$$

for a given $\varepsilon_{0}$, we have $\delta>0$ s.t

$$
(1-\delta) *(1-\delta)>1-\varepsilon_{0} \text { and } \delta \diamond \delta<\varepsilon_{0}
$$

then we define for $t_{1}>0$,

$$
\begin{aligned}
& K_{1}=\left\{(n, k, l) \in \mathbf{N} \times \mathbf{N} \times \mathbf{N}: \mu\left(T\left(x_{n k l}\right)-\xi_{1}, \frac{t_{1}}{2}\right) \leq 1-\delta \text { or } \nu\left(T\left(x_{n k l}\right)-\xi_{1}, \frac{t_{1}}{2}\right)>\delta\right\}, \\
& K_{2}=\left\{(n, k, l) \in \mathbf{N} \times \mathbf{N} \times \mathbf{N}: \mu\left(T\left(x_{n k l}\right)-\xi_{2}, \frac{t_{1}}{2}\right) \leq 1-\delta \text { or } \nu\left(T\left(x_{n k l}\right)-\xi_{2}, \frac{t_{1}}{2}\right)>\delta\right\},
\end{aligned}
$$

since

$$
I_{3}^{(\mu, \nu)} \lim x_{n k l}=\xi_{1} \text { and } I_{3}^{(\mu, \nu)} \lim x_{n k l}=\xi_{2}
$$


$\Rightarrow K_{1} \in I_{3}$ and $K_{2} \in I_{3} \forall t_{1}>0$

Let $K=K_{1} \cup K_{2}, \Rightarrow K \in I_{3}$ and $K^{c} \in F\left(I_{3}\right)$.

If, $(p, q, r) \in K^{c}$, then

$$
\begin{gathered}
\mu\left(\xi_{1}-\xi_{2}, t_{1}\right) \geq \mu\left(T x_{p q r}-\xi_{1}, \frac{t_{1}}{2}\right) * \mu\left(T x_{p q r}-\xi_{2}, \frac{t_{1}}{2}\right) \\
>(1-\delta) *(1-\delta) \\
>1-\varepsilon_{0}
\end{gathered}
$$

$\varepsilon_{0}>0$ being arbitrary,

$$
\Rightarrow \mu\left(\xi_{1}-\xi_{2}, t_{1}\right)=1 \forall t_{1}>0 \Rightarrow \xi_{1}=\xi_{2} .
$$

Similarly it can be proved that,

$$
\nu\left(\xi_{1}-\xi_{2}, t_{1}\right)<\varepsilon_{0} \forall t_{1}>0 \Rightarrow \xi_{1}=\xi_{2} .
$$

Thereby one can conclude that,

$$
I_{3}^{(\mu, \nu)} \lim x_{n k l}=\xi \text { is unique. }
$$

Theorem 3.2: Let $x=\left(x_{n k l}\right)$ be a triple sequence in ${ }_{3} \mathcal{S}_{(\mu, \nu)}^{I}(T)$, then following assertions are equivalent:

(a) If $I_{3}^{(\mu, \nu)} \lim x_{n k l}=\xi$,

(b) $\left\{(n, k, l) \in \mathbf{N} \times \mathbf{N} \times \mathbf{N}: \mu\left(T\left(x_{n k l}\right)-\xi, t_{1}\right) \leq 1-\varepsilon_{0}\right.$ or $\nu\left(T\left(x_{n k l}\right)-\right.$ $\left.\left.\xi, t_{1}\right) \geq \varepsilon_{0}\right\} \in I_{3}$

(c) $\left\{(n, k, l) \in \mathbf{N} \times \mathbf{N} \times \mathbf{N}: \mu\left(T\left(x_{n k l}\right)-\xi, t_{1}\right)>1-\varepsilon_{0}\right.$ or $\nu\left(T\left(x_{n k l}\right)-\right.$ $\left.\left.\xi, t_{1}\right)<\varepsilon_{0}\right\} \in F\left(I_{3}\right)$

(d) $I_{3} \lim \mu\left(T\left(x_{n k l}\right)-\xi, t_{1}\right)=1$ and $I_{3} \lim \nu\left(T\left(x_{n k l}\right)-\xi, t_{1}\right)=0$.

Theorem 3.3: The spaces ${ }_{3} \mathcal{S}_{(\mu, \nu)}^{I}(T)$ and ${ }_{3} \mathcal{S}_{0(\mu, \nu)}^{I}(T)$ are linear spaces.

Proof: We prove the linearity of the space ${ }_{3} \mathcal{S}_{(\mu, \nu)}^{I}(T)$. For other space it directly follows. 
Let $x=\left(x_{n k l}\right), y=\left(y_{n k l}\right) \in{ }_{3} \mathcal{S}_{(\mu, \nu)}^{I}(T)$ and $\alpha, \beta$ be non-zero scalars and $\epsilon_{o}>0$ be given, then

$$
\begin{gathered}
A_{1}=\left\{(n, k, l) \in \mathbf{N} \times \mathbf{N} \times \mathbf{N}: \mu\left(T\left(x_{n k l}\right)-\xi_{1}, \frac{t_{1}}{2|\alpha|}\right) \leq 1-\varepsilon_{0}\right. \\
\text { or } \left.\nu\left(T\left(x_{n k l}\right)-\xi_{1}, \frac{t_{1}}{2|\alpha|}\right) \geq \varepsilon_{0}\right\} \in I_{3}, \\
A_{2}=\left\{(n, k, l) \in \mathbf{N} \times \mathbf{N} \times \mathbf{N}: \mu\left(T\left(y_{n k l}\right)-\xi_{2}, \frac{t_{1}}{2|\beta|}\right) \leq 1-\varepsilon_{0}\right. \\
\text { or } \left.\nu\left(T\left(y_{n k l}\right)-\xi_{2}, \frac{t_{1}}{2|\beta|}\right) \geq \varepsilon_{0}\right\} \in I_{3}, \\
A_{1}^{c}=\left\{(n, k, l) \in \mathbf{N} \times \mathbf{N} \times \mathbf{N}: \mu\left(T\left(x_{n k l}\right)-\xi_{1}, \frac{t_{1}}{2|\alpha|}\right)>1-\varepsilon_{0}\right. \\
\text { or } \left.\nu\left(T\left(x_{n k l}\right)-\xi_{1}, \frac{t_{1}}{2|\alpha|}\right)<\varepsilon_{0}\right\} \in \mathcal{F}\left(I_{3}\right) . \\
A_{2}^{c}=\left\{(n, k, l) \in \mathbf{N} \times \mathbf{N} \times \mathbf{N}: \mu\left(T\left(y_{n k l}\right)-\xi_{2}, \frac{t_{1}}{2|\beta|}\right)>1-\varepsilon_{0}\right. \\
\text { or } \left.\nu\left(T\left(y_{n k l}\right)-\xi_{2}, \frac{t_{1}}{2|\beta|}\right)<\varepsilon_{0}\right\} \in \mathcal{F}\left(I_{3}\right) .
\end{gathered}
$$

Define $A_{3}=A_{1} \cup A_{2}$, so that $A_{3} \in I_{3} \Rightarrow A_{3}^{c} \in \mathcal{F}\left(I_{3}\right)$ is non-empty. Now we show that for each $\left(x_{n k l}\right),\left(y_{n k l}\right) \in{ }_{3} \mathcal{S}_{(\mu, \nu)}^{I}(T)$.

$$
\begin{gathered}
A_{3}^{c} \subset\left\{(n, k, l) \in \mathbf{N} \times \mathbf{N} \times \mathbf{N}: \mu\left(\left(\alpha T\left(x_{n k l}\right)+\beta T\left(y_{n k l}\right)\right)-\left(\alpha \xi_{1}+\beta \xi_{2}\right), t_{1}\right)>1-\varepsilon_{0}\right. \\
\text { or } \left.\nu\left(\left(\alpha T\left(x_{n k l}\right)+\beta T\left(y_{n k l}\right)\right)-\left(\alpha \xi_{1}+\beta \xi_{2}\right), t_{1}\right)<\varepsilon_{0}\right\} .
\end{gathered}
$$

Let $(p, q, r) \in A_{3}^{c}$. In this case

$$
\mu\left(T\left(x_{p q r}\right)-\xi_{1}, \frac{t_{1}}{2|\alpha|}\right)>1-\varepsilon_{0} \text { or } \nu\left(T\left(x_{p q r}\right)-\xi_{1}, \frac{t_{1}}{2|\alpha|}\right)<\varepsilon_{0}
$$

and

$$
\mu\left(T\left(y_{p q r}\right)-\xi_{2}, \frac{t_{1}}{2|\beta|}\right)>1-\varepsilon_{0} \text { or } \nu\left(T\left(y_{p q r}\right)-\xi_{2}, \frac{t_{1}}{2|\beta|}\right)<\varepsilon_{0} .
$$


We have

$$
\begin{gathered}
\mu\left(\left(\alpha T\left(x_{p q r}\right)+\beta T\left(y_{p q r}\right)\right)-\left(\alpha \xi_{1}+\beta \xi_{2}\right), t_{1}\right) \\
\geq \mu\left(\alpha T\left(x_{p q r}\right)-\alpha \xi_{1}, \frac{t_{1}}{2}\right) * \mu\left(\beta T\left(x_{p q r}\right)-\beta \xi_{2}, \frac{t_{1}}{2}\right) \\
=\mu\left(T\left(x_{p q r}\right)-\xi_{1}, \frac{t_{1}}{2|\alpha|}\right) * \mu\left(T\left(x_{p q r}\right)-\xi_{2}, \frac{t_{1}}{2|\beta|}\right) \\
>\left(1-\varepsilon_{0}\right) *\left(1-\epsilon_{o}\right)=1-\varepsilon_{0} .
\end{gathered}
$$

and

$$
\begin{gathered}
\nu\left(\left(\alpha T\left(x_{p q r}\right)+\beta T\left(y_{p q r}\right)\right)-\left(\alpha \xi_{1}+\beta \xi_{2}\right), t_{1}\right) \\
\leq \nu\left(\alpha T\left(x_{p q r}\right)-\alpha \xi_{1}, \frac{t_{1}}{2}\right) \diamond \nu\left(\beta T\left(x_{p q r}\right)-\beta \xi_{2}, \frac{t_{1}}{2}\right) \\
=\mu\left(T\left(x_{p q r}\right)-\xi_{1}, \frac{t_{1}}{2|\alpha|}\right) \diamond \mu\left(T\left(x_{p q r}\right)-\xi_{2}, \frac{t_{1}}{2|\beta|}\right) \\
<\varepsilon_{0} \diamond \varepsilon_{0}=\varepsilon_{0} .
\end{gathered}
$$

Hence,

$A_{3}^{c} \subset\left\{(n, k, l) \in \mathbf{N} \times \mathbf{N} \times \mathbf{N}: \mu\left(\left(\alpha T\left(x_{n k l}\right)+\beta T\left(y_{n k l}\right)\right)-\left(\alpha \xi_{1}+\beta \xi_{2}\right), t_{1}\right)>1-\varepsilon_{0}\right.$

$$
\text { or } \left.\nu\left(\left(\alpha T\left(x_{n k l}\right)+\beta T\left(y_{n k l}\right)\right)-\left(\alpha \xi_{1}+\beta \xi_{2}\right), t_{1}\right)<\varepsilon_{0}\right\} \text {. }
$$

Hence ${ }_{3} \mathcal{S}_{(\mu, \nu)}^{I}(T)$ is a linear space.

Theorem 3.4: $\quad$ Let $x=\left(x_{n k l}\right)$ be a triple sequence in ${ }_{3} \mathcal{S}_{(\mu, \nu)}^{I}(T)$ such that $(\mu, \nu)_{3} \lim x_{n k l}=\xi$, then $I_{3}^{(\mu, \nu)} \lim x_{n k l}=\xi$.

Proof: Let $(\mu, \nu)_{3} \lim x_{n k l}=\xi$, and $\varepsilon_{0}>0$ be given then $\forall t_{1}>0, \exists$ $(p, q, r) \in \mathbf{N} \times \mathbf{N} \times \mathbf{N}$ s.t.

$$
\mu\left(T\left(x_{n k l}\right)-\xi\right)>1-\epsilon_{o} \text { and } \nu\left(T\left(x_{n k l}\right)-\xi\right)<\varepsilon_{0}
$$

$\forall(n, k, l) \geq(p, q, r)$.

Therefore, we get

$$
\begin{aligned}
B=\left\{(n, k, l) \in \mathbf{N} \times \mathbf{N} \times \mathbf{N}: \mu\left(\left(T\left(x_{n k l}\right)-\xi, t_{1}\right) \leq 1-\varepsilon_{0} \text { or } \nu\left(\left(T\left(x_{n k l}\right)-\xi, t_{1}\right) \geq \varepsilon_{0}\right\}\right.\right. \\
\subseteq\left\{\left(p^{\prime}, q^{\prime}, r^{\prime}\right) \in \mathbf{N} \times \mathbf{N} \times \mathbf{N}: p^{\prime}<p-1, q^{\prime}<q-1, r^{\prime}<r-1\right\}
\end{aligned}
$$


But, $I_{3}$ being admissible $\Rightarrow B \in I_{3}$

Hence,

$$
I_{3}^{(\mu, \nu)} \lim x_{n k l}=\xi
$$

Theorem 3.5: Let $x=\left(x_{n k l}\right)$ be a triple sequence in $\quad{ }_{3} \omega$. If $y=\left(y_{n k l}\right)$ in ${ }_{3} \omega$ is a $I_{3}^{(\mu, \nu)}$ convergent sequence s.t.

$$
\left\{(n, k, l) \in \mathbf{N} \times \mathbf{N} \times \mathbf{N}: x_{n k l} \neq y_{n k l}\right\} \in I_{3},
$$

then $x$ is also $I_{3}^{(\mu, \nu)}$ convergent.

Proof: Consider the set, $\left\{(n, k, l) \in \mathbf{N} \times \mathbf{N} \times \mathbf{N}: x_{n k l} \neq y_{n k l}\right\} \in I_{3}$ and $I_{3}^{(\mu, \nu)} \lim y_{n k l}=\xi$ then $0<\varepsilon_{0}<1 \forall t_{1}>0$, we get

$$
\begin{gathered}
\left\{(n, k, l) \in \mathbf{N} \times \mathbf{N} \times \mathbf{N}: \mu\left(\left(T\left(y_{n k l}\right)-\xi, t_{1}\right) \leq 1-\epsilon_{o}\right.\right. \\
\text { or } \nu\left(\left(T\left(y_{n k l}\right)-\xi, t_{1}\right) \geq \varepsilon_{0}\right\} \in I_{3} .
\end{gathered}
$$

$0<\varepsilon_{0}<1 \forall t_{1}>0$,

$$
\begin{gathered}
\left\{(n, k, l) \in \mathbf{N} \times \mathbf{N} \times \mathbf{N}: \mu\left(\left(T\left(x_{n k l}\right)-\xi, t_{1}\right) \leq 1-\varepsilon_{0}\right.\right. \\
\text { or } \nu\left(\left(T\left(x_{n k l}\right)-\xi, t_{1}\right) \geq \varepsilon_{0}\right\} . \\
\subseteq\left\{(n, k, l) \in \mathbf{N} \times \mathbf{N} \times \mathbf{N}: x_{n k l} \neq y_{n k l}\right\} \\
\cup\left\{(n, k, l) \in \mathbf{N} \times \mathbf{N} \times \mathbf{N}: \mu\left(\left(T\left(y_{n k l}\right)-\xi, t_{1}\right) \leq 1-\varepsilon_{0}\right.\right. \\
\quad \text { or } \nu\left(\left(T\left(y_{n k l}\right)-\xi, t_{1}\right) \geq \varepsilon_{0}\right\}
\end{gathered}
$$

Right hand side of (1) is an element of $I_{3}$, therefore we get

$$
\begin{gathered}
\left\{(n, k, l) \in \mathbf{N} \times \mathbf{N} \times \mathbf{N}: \mu\left(\left(T\left(x_{n k l}\right)-\xi, t_{1}\right) \leq 1-\varepsilon_{0}\right.\right. \\
\quad \text { or } \nu\left(\left(T\left(x_{n k l}\right)-\xi, t_{1}\right) \geq \varepsilon_{0}\right\} \in I_{3} .
\end{gathered}
$$

Theorem 3.6: If $x=\left(x_{n k l}\right) \in{ }_{3} \mathcal{S}_{(\mu, \nu)}^{I}(T)$ is a Cauchy sequence with respect to intuitionistic norm $(\mu, \nu)$ then it is $I_{3}^{(\mu, \nu)}$-Cauchy with respect to same norm. 
Proof: The proof is straight forward thus omitted.

Theorem 3.7: If $x=\left(x_{n k l}\right) \in{ }_{3} \mathcal{S}_{(\mu, \nu)}^{I}(T)$ is a Cauchy sequence with respect to intuitionistic norm $(\mu, \nu)$ then the sequence $x=\left(x_{n k l}\right)$ has a subsequence which is an ordinary Cauchy sequence with respect to the same norm.

Proof: The proof is straight forward, thus omitted.

Theorem 3.8: Every open ball ${ }_{3} \mathcal{B}_{x}\left(\delta, t_{1}\right)(T)$ is an open set in ${ }_{3} \mathcal{S}_{(\mu, \nu)}^{I}(T)$.

Proof: Let ${ }_{3} \mathcal{B}_{x}\left(\delta, t_{1}\right)(T)$ be an open ball with radius $\delta$, centered at $x$ with respect to $t_{1}$.

i.e

$$
\begin{gathered}
{ }_{3} \mathcal{B}_{x}\left(\delta, t_{1}\right)(T)=\left\{y=\left(y_{n k l}\right) \in{ }_{3} \omega:\left\{(n, k, l) \in \mathbf{N} \times \mathbf{N} \times \mathbf{N}: \mu\left(T\left(x_{n k l}\right)\right.\right.\right. \\
\left.\left.\left.-T\left(y_{n k l}\right), t_{1}\right)>1-\delta \text { and } \nu\left(T\left(x_{n k l}\right)-T\left(y_{n k l}\right), t_{1}\right)<\delta\right\} \in I_{3}\right\} .
\end{gathered}
$$

Let $y \in{ }_{3} \mathcal{B}_{x}^{c}\left(\delta, t_{1}\right)(T)$.

Then, $\mu\left(T\left(x_{n k l}\right)-T\left(y_{n k l}\right), t_{1}\right)>1-\delta$ and $\nu\left(T\left(x_{n k l}\right)-T\left(y_{n k l}\right), t_{1}\right)<\delta$.

Since, $\mu\left(T\left(x_{n k l}\right)-T\left(y_{n k l}\right), t_{1}\right)>1-\delta, \exists 0<t_{o}<t_{1}$

s.t.,

$$
\mu\left(T\left(x_{n k l}\right)-T\left(y_{n k l}\right), t_{o}\right)>1-\delta \text { and } \nu\left(T\left(x_{n k l}\right)-T\left(y_{n k l}\right), t_{o}\right)<\delta .
$$

Now, Consider

$$
\delta_{o}=\mu\left(T\left(x_{n k l}\right)-T\left(y_{n k l}\right), t_{o}\right), \text { we have } \delta_{o}>1-\delta
$$

there exists,

$$
t_{2} \in(0,1) \quad \text { such that } \delta_{o}>1-t_{2}>1-\delta
$$

For $\delta_{o}>1-t_{2}$, we have $\delta_{1}, \delta_{2} \in(0,1)$ such that $\delta_{o} * \delta_{1}>1-t_{2}$ and $\left(1-\delta_{o}\right) \diamond\left(1-\delta_{2}\right) \leq t_{2}$.

let $\delta_{3}=\max \left\{\delta_{1}, \delta_{2}\right\}$.

Here we consider the open ball ${ }_{3} \mathcal{B}_{y}^{c}\left(1-\delta_{3}, t_{1}-t_{o}\right)(T)$ and we show that

$$
{ }_{3} \mathcal{B}_{y}^{c}\left(1-\delta_{3}, t_{1}-t_{o}\right)(T) \subset{ }_{3} \mathcal{B}_{x}^{c}\left(\delta, t_{1}\right)(T) .
$$


Let,

$$
z=\left(z_{n k l}\right) \in{ }_{3} \mathcal{B}_{y}^{c}\left(1-\delta_{3}, t_{1}-t_{o}\right)(T)
$$

then

$$
\begin{gathered}
\mu\left(T\left(y_{n k l}\right)-T\left(z_{n k l}\right), t_{1}-t_{o}\right)>\delta_{3} \\
\text { and } \nu\left(T\left(y_{n k l}\right)-T\left(z_{n k l}\right), t_{1}-t_{o}\right)<1-\delta_{3} .
\end{gathered}
$$

Therefore,

$$
\begin{gathered}
\mu\left(T\left(x_{n k l}\right)-T\left(z_{n k l}\right), t_{1}\right) \\
\geq \mu\left(T\left(x_{n k l}\right)-T\left(y_{n k l}\right), t_{o}\right) * \mu\left(T\left(y_{n k l}\right)-T\left(z_{n k l}\right), t_{1}-t_{o}\right) \\
\geq\left(\delta_{o} * \delta_{3}\right) \geq\left(\delta_{o} * \delta_{1}\right) \geq\left(1-t_{2}\right) \geq(1-\delta)
\end{gathered}
$$

and

$$
\begin{gathered}
\nu\left(T\left(x_{n k l}\right)-T\left(z_{n k l}\right), t_{1}\right) \\
\leq \nu\left(T\left(x_{n k l}\right)-T\left(y_{n k l}\right), t_{o}\right) \diamond \nu\left(T\left(y_{n k l}\right)-T\left(z_{n k l}\right), t_{1}-t_{o}\right) \\
\leq\left(1-\delta_{o}\right) \diamond\left(1-\delta_{3}\right) \leq\left(1-\delta_{o}\right) \diamond\left(1-\delta_{2}\right) \leq t_{2} \geq \delta .
\end{gathered}
$$

Thus

$$
z \in{ }_{3} \mathcal{B}_{x}^{c}\left(\delta, t_{1}\right)(T)
$$

hence

$$
{ }_{3} \mathcal{B}_{y}^{c}\left(1-\delta_{3}, t_{1}-t_{o}\right)(T) \subset{ }_{3} \mathcal{B}_{x}^{c}\left(\delta, t_{1}\right)(T)
$$

Remark: ${ }_{3} \mathcal{S}_{(\mu, \nu)}^{I}(T)$ is an IFNS.

Consider the set

$$
\begin{gathered}
{ }_{3} \tau_{(\mu, \nu)}^{I}(T)=\left\{\mathcal{A} \subset{ }_{3} \mathcal{S}_{(\mu, \nu)}^{I}(T): \forall x \in \mathcal{A} \exists t_{1}>0\right. \\
\text { and } \left.0<\delta<1 \text { s.t. }{ }_{3} \mathcal{B}_{x}\left(\delta, t_{1}\right)(T) \subset \mathcal{A}\right\} .
\end{gathered}
$$

$\Rightarrow{ }_{3} \tau_{(\mu, \nu)}^{I}(T)$ is clearly a topology on ${ }_{3} \mathcal{S}_{(\mu, \nu)}^{I}(T)$.

Theorem 3.9: The topology ${ }_{3} \tau_{(\mu, \nu)}^{I}(T)$ on ${ }_{3} \mathcal{S}_{0(\mu, \nu)}^{I}(T)$ is first countable.

Proof: $\left\{{ }_{3} \mathcal{B}_{x}\left(\frac{1}{k}, \frac{1}{k}\right)(T): k=1,2,3, \ldots\right\}$ forms a local base at $x$ therefore the topology ${ }_{3} \tau_{(\mu, \nu)}^{I}(T)$ on ${ }_{3} \mathcal{S}_{0(\mu, \nu)}^{I}(T)$ is first countable. 
Theorem 3.10: ${ }_{3} \mathcal{S}_{(\mu, \nu)}^{I}(T)$ and ${ }_{3} S_{0(\mu, \nu)}^{I}(T)$ are Hausdorff spaces.

Proof: We establish the result for the space ${ }_{3} \mathcal{S}_{(\mu, \nu)}^{I}(T)$.

The result follows directly for ${ }_{3} \mathcal{S}_{0(\mu, \nu)}^{I}(T)$.

Let,

$$
x=\left(x_{n k l}\right), y=\left(y_{n k l}\right) \in{ }_{3} \mathcal{S}_{(\mu, \nu)}^{I}(T) \text { s.t. }\left(x_{n k l}\right) \neq\left(y_{n k l}\right) .
$$

Then

$$
0<\mu\left(T\left(x_{n k l}\right)-T\left(y_{n k l}\right), t_{1}\right)<1 \text { and } 0<\nu\left(T\left(x_{n k l}\right)-T\left(y_{n k l}\right), t_{1}\right)<1 \text {. }
$$

consider, $\delta_{1}=\mu\left(T\left(x_{n k l}\right)-T\left(y_{n k l}\right), t_{1}\right), \delta_{2}=\nu\left(T\left(x_{n k l}\right)-T\left(y_{n k l}\right), t_{1}\right)$ and $\delta=\max \left\{\delta_{1}, 1-\delta_{2}\right\}$.

$\forall \delta_{o} \in(\delta, 1) \exists \delta_{3}$ and $\delta_{4}$ s.t. $\delta_{3} * \delta_{3} \geq \delta_{o}$ and

$$
\left(1-\delta_{4}\right) \diamond\left(1-\delta_{4}\right) \leq\left(1-\delta_{o}\right) .
$$

Again for, $\delta_{5}=\max \left\{\delta_{3}, \delta_{4}\right\}$ and we consider ${ }_{3} \mathcal{B}_{x}\left(1-\delta_{5}, \frac{t_{1}}{2}\right)$ and ${ }_{3} \mathcal{B}_{y}(1-$ $\left.\delta_{5}, \frac{t_{1}}{2}\right)$.

Clearly,

$$
{ }_{3} \mathcal{B}_{x}^{c}\left(1-\delta_{5}, \frac{t_{1}}{2}\right) \cap{ }_{3} \mathcal{B}_{y}^{c}\left(1-\delta_{5}, \frac{t_{1}}{2}\right)=\emptyset .
$$

For, if there exists

$$
z=\left(z_{n k l}\right) \in{ }_{3} \mathcal{B}_{x}^{c}\left(1-\delta_{5}, \frac{t_{1}}{2}\right) \cap{ }_{3} \mathcal{B}_{y}^{c}\left(1-\delta_{5}, \frac{t_{1}}{2}\right)
$$

then

$$
\begin{gathered}
\delta_{1}=\mu\left(T\left(x_{n k l}\right)-T\left(y_{n k l}\right), t_{1}\right) \\
\geq \mu\left(T\left(x_{n k l}\right)-T\left(z_{n k l}\right), \frac{t_{1}}{2}\right) * \mu\left(T\left(z_{n k l}\right)-T\left(y_{n k l}\right), \frac{t_{1}}{2}\right) \\
\geq \delta_{5} * \delta_{5} \geq \delta_{3} * \delta_{3} \geq \delta_{o}>\delta_{1}
\end{gathered}
$$

and

$$
\begin{gathered}
\delta_{2}=\nu\left(T\left(x_{n k l}\right)-T\left(y_{n k l}\right), t_{1}\right) \\
\leq \nu\left(T\left(x_{n k l}\right)-T\left(z_{n k l}\right), \frac{t_{1}}{2}\right) \diamond \nu\left(T\left(z_{n k l}\right)-T\left(y_{n k l}\right), \frac{t_{1}}{2}\right) \\
\leq\left(1-\delta_{5}\right) \diamond\left(1-\delta_{5}\right) \\
\leq\left(1-\delta_{4}\right) \diamond\left(1-\delta_{4}\right) \leq\left(1-\delta_{o}\right)<\delta_{2}
\end{gathered}
$$


which is not possible.

Therefore, ${ }_{3} \mathcal{S}_{(\mu, \nu)}^{I}(T)$ is Hausdorff.

Theorem 3.11: ${ }_{3} \mathcal{S}_{(\mu, \nu)}^{I}(T)$ is an IFNS and ${ }_{3} \tau_{(\mu, \nu)}^{I}(T)$ is a topology on ${ }_{3} \mathcal{S}_{(\mu, \nu)}^{I}(T)$.

Then $\exists$ a sequence $\left(x_{n k l}\right) \in{ }_{3} \mathcal{S}_{(\mu, \nu)}^{I}(T)$, such that $x_{n k l} \rightarrow x$ iff $\mu\left(\left(T\left(x_{n k l}\right)-\right.\right.$ $\left.T(x), t_{1}\right) \rightarrow 1$ and $\nu\left(T\left(x_{n k l}\right)-T(x), t_{1}\right) \rightarrow 0$ as $n, k, l \rightarrow \infty$.

Proof: Let $t_{1}>0$, let $x_{n k l} \rightarrow x$ and $0<\delta<1, \exists(p, q, r) \in \mathbf{N} \times \mathbf{N} \times \mathbf{N}$ s.t.,

$$
\begin{gathered}
\left(x_{n k l}\right) \in{ }_{3} \mathcal{B}_{x}\left(\delta, t_{1}\right)(T) \text { for all } n \geq p, k \geq q, l \geq r, \\
{ }_{3} \mathcal{B}_{x}\left(\delta, t_{1}\right)(T)=\left\{(n, k, l) \in \mathbf{N} \times \mathbf{N} \times \mathbf{N}: \mu\left(T\left(x_{n k l}\right)-T(x), t_{1}\right)\right\}>1-\delta \\
\text { and } \left.\nu\left(T\left(x_{n k l}\right)-T(x), t_{1}\right)<\delta\right\} \in I_{3} .
\end{gathered}
$$

such that,

$$
{ }_{3} \mathcal{B}_{x}^{c}\left(\delta, t_{1}\right)(T) \in \mathcal{F}\left(I_{3}\right) .
$$

Then,

$$
1-\mu\left(T\left(x_{n k l}\right)-T(x), t_{1}\right)<\delta \text { and } \nu\left(T\left(x_{n k l}\right)-T(x), t_{1}\right)<\delta .
$$

Hence,

$\mu\left(T\left(x_{n k l}\right)-T(x), t_{1}\right) \rightarrow 1$ and $\nu\left(T\left(x_{n k l}\right)-T(x), t_{1}\right) \rightarrow 0$, as $n, k, l \rightarrow \infty$.

Conversely,

$\mu\left(T\left(x_{n k l}\right)-T(x), t_{1}\right) \rightarrow 1$ and $\nu\left(T\left(x_{n k l}\right)-T(x), t_{1}\right) \rightarrow 0$ as $n, k, l \rightarrow \infty$,

then for,

$$
0<\delta<1, \exists(p, q, r) \in \mathbf{N} \times \mathbf{N} \times \mathbf{N}
$$

s.t.,

$1-\mu\left(T\left(x_{n k l}\right)-T(x), t_{1}\right)<\delta$ and $\nu\left(T\left(x_{n k l}\right)-T(x), t_{1}\right)<\delta, \quad \forall n \geq p, k \geq q, l \geq r$.

It follows that,

$\mu\left(T\left(x_{n k l}\right)-T(x), t_{1}\right)>1-\delta$ and $\nu\left(T\left(x_{n k l}\right)-T(x), t_{1}\right)<\delta \quad \forall n \geq p, k \geq q, l \geq r$. 
Thus,

$$
\left(x_{n k l}\right) \in{ }_{3} \mathcal{B}_{x}^{c}\left(\delta, t_{1}\right)(T) \text { for all } n \geq p, k \geq q, l \geq r
$$

hence,

$$
x_{n k l} \rightarrow x
$$

Theorem.3.12: A triple sequence $x=\left(x_{n k l}\right) \in{ }_{3} S_{(\mu, \nu)}^{I}(T)$ is $I$-convergent iff for every $\varepsilon_{0}>0, t_{1}>0$ there exists numbers $p=p\left(x, \varepsilon_{0}, t_{1}\right), q=$ $q\left(x, \varepsilon_{0}, t_{1}\right)$ and $r=r\left(x, \varepsilon_{0}, t_{1}\right)$ such that,

$$
\begin{gathered}
\left\{(p, q, r) \in \mathbf{N} \times \mathbf{N} \times \mathbf{N}: \mu\left(T\left(x_{p q r}\right)-\xi, \frac{t_{1}}{2}\right)>1-\varepsilon_{0}\right. \\
\left.\quad \text { or } \nu\left(T\left(x_{p q r}\right)-\xi, \frac{t_{1}}{2}\right)<\varepsilon_{0}\right\} \in \mathcal{F}\left(I_{3}\right) .
\end{gathered}
$$

Proof: Let $I_{3}^{(\mu, \nu)} \lim x=\xi$ and let $\varepsilon_{0}>0$ and $t_{1}>0$.For $\varepsilon_{0}>0$ to be given, choose $t_{2}>0$

s.t.,

$$
\left(1-\varepsilon_{0}\right) *\left(1-\varepsilon_{0}\right)>1-t_{2} \text { and } \varepsilon_{0} \diamond \varepsilon_{0}<t_{2} .
$$

we get,

$$
\begin{gathered}
x \in{ }_{3} S_{(\mu, \nu)}^{I}(T), \\
P=\left\{(n, k, l) \in \mathbf{N} \times \mathbf{N} \times \mathbf{N}: \mu\left(T\left(x_{n k l}\right)-\xi, \frac{t_{1}}{2}\right) \leq 1-\varepsilon_{0}\right. \\
\text { or } \left.\nu\left(T\left(x_{n k l}\right)-\xi, \frac{t_{1}}{2}\right) \geq \varepsilon_{0}\right\} \in I_{3},
\end{gathered}
$$

This implies

$$
\begin{gathered}
P^{c}=\left\{(n, k, l) \in \mathbf{N} \times \mathbf{N} \times \mathbf{N}: \mu\left(T\left(x_{n k l}\right)-\xi, \frac{t_{1}}{2}\right)>1-\varepsilon_{0}\right. \\
\text { or } \left.\nu\left(T\left(x_{n k l}\right)-\xi, \frac{t_{1}}{2}\right)<\varepsilon_{0}\right\} \in \mathcal{F}\left(I_{3}\right) .
\end{gathered}
$$

Conversely, let $p, q, r \in P$.

Then

$$
\mu\left(T\left(x_{p q r}\right)-\xi, \frac{t_{1}}{2}\right)>1-\varepsilon_{0} \text { or } \nu\left(T\left(x_{p q r}\right)-\xi, \frac{t_{1}}{2}\right)<\varepsilon_{0} .
$$

Now, we show that $\exists p=p\left(x, \varepsilon_{0}, t_{1}\right)$ and $q=q\left(x, \varepsilon_{0}, t_{1}\right)$ and $r=r\left(x, \varepsilon_{0}, t_{1}\right)$ such that,

$\left\{(n, k, l): \mu\left(T\left(x_{n k l}\right)-T\left(x_{p q r}\right), t_{1}\right) \leq 1-t_{2}\right.$ or $\left.\nu\left(T\left(x_{n k l}\right)-T\left(x_{p q r}\right), t_{1}\right) \geq t_{2}\right\} \in I_{3}$. 
Thus, for each $x \in{ }_{3} S_{(\mu, \nu)}^{I}(T)$ we consider

$$
\begin{aligned}
Q= & \left\{(n, k, l): \mu\left(T\left(x_{n k l}\right)-T\left(x_{p q r}\right), t_{1}\right) \leq 1-t_{2}\right. \\
& \text { or } \left.\nu\left(T\left(x_{n k l}\right)-T\left(x_{p q r}\right), t_{1}\right) \geq t_{2}\right\} \in I_{3} .
\end{aligned}
$$

Now we show that $Q \subset P$.

Let $Q \not \subset P$ then $\exists\left(n^{\prime}, k^{\prime}, l^{\prime}\right) \in Q$ and $\left(n^{\prime}, k^{\prime}, l^{\prime}\right) \notin P$.

Therefore we get,

$$
\mu\left(T\left(x_{n^{\prime}, k^{\prime}, l^{\prime}}\right)-T\left(x_{p q r}\right), t_{1}\right) \leq 1-t_{2} \text { or } \mu\left(T\left(x_{n^{\prime}, k^{\prime}, l^{\prime}}\right)-\xi, \frac{t_{1}}{2}\right)>1-\varepsilon_{0} .
$$

particularly,

$$
\mu\left(T\left(x_{p q r}\right)-\xi, \frac{t_{1}}{2}\right)>1-\varepsilon_{0} .
$$

moreover we get,

$$
\begin{gathered}
1-t_{2} \geq \mu\left(T\left(x_{n^{\prime}, k^{\prime}, l^{\prime}}\right)-T\left(x_{p q r}\right), t_{1}\right) \\
\geq \mu\left(T\left(x_{n^{\prime}, k^{\prime}, l^{\prime}}\right)-\xi, \frac{t_{1}}{2}\right) * \mu\left(T\left(x_{p q r}\right)-\xi, \frac{t_{1}}{2}\right) \\
\geq\left(1-\varepsilon_{0}\right) *\left(1-\epsilon_{o}\right)>1-t_{2},
\end{gathered}
$$

which is not possible.

On the other hand,

$$
\nu\left(T\left(x_{n^{\prime}, k^{\prime}, l^{\prime}}\right)-T\left(x_{p q r}\right), t_{1}\right) \geq t_{2} \text { or } \nu\left(T\left(x_{n^{\prime}, k^{\prime}, l^{\prime}}\right)-\xi, \frac{t_{1}}{2}\right)<\varepsilon_{0}
$$

particularly,

$$
\nu\left(T\left(x_{p q r}\right)-\xi, \frac{t_{1}}{2}\right)<\varepsilon_{0} .
$$

Therefore we get,

$$
\begin{gathered}
t_{2} \leq \nu\left(T\left(x_{n^{\prime}, k^{\prime}, l^{\prime}}\right)-T\left(x_{p q r}\right), t_{1}\right) \\
\leq \nu\left(T\left(x_{n^{\prime}, k^{\prime}, l^{\prime}}\right)-\xi, \frac{t_{1}}{2}\right) \diamond \nu\left(T\left(x_{p q r}\right)-\xi, \frac{t_{1}}{2}\right) \\
\leq \varepsilon_{0} \diamond \varepsilon_{0}<t_{2},
\end{gathered}
$$

which is not possible.

Hence,

$$
Q \subset P
$$

and we have,

$$
P \in I \Rightarrow Q \in I \text {. }
$$




\section{Acknowledgment}

The authors would like to thank the referees and the editor for their careful reading and their valuable comments.

\section{References}

[1] A. Esi, "On some triple almost lacunary sequence spaces defined by Orlicz functions", Research and Reviews: Discrete Mathematical Structures, vol. 1, no. 2, pp. 16-25, 2014.

[2] A. Esi and C. Necdet, "Almost convergence of triple sequences", Global journal of mathematical analysis, vol. 2, no. 1, pp. 6-10, 2014.

[3] A. Sahiner, M. Gurdal, and K. Duden, "Triple sequences and their statistical convergence", Selçuk Journal of Applied Mathematics, vol. 8, no. 2, pp. 49-55, 2007.

[4] A. Sahiner and B. C. Tripathy, "Some I-related properties of Triple sequences", Selçuk Journal of Applied Mathematics, vol. 9, no. 2, pp. 9-18, 2008.

[5] B. C. Das, "Some I-convergent triple sequence spaces defined by a sequence of modulus function", Proyecciones (Antofagasta), vol. 36, no. 1, pp. 117-130, 2017.

[6] B. C. Tripathy, "Statistically convergent double sequences", Tamkang Journal of Mathematics, vol.34, no. 3, pp. 231-237, 2003.

[7] B. C. Tripathy and S. Mahanta, "On I-acceleration convergence of sequences", Journal of the Franklin Institute, vol. 347, no. 3, pp. 591-598, 2010.

[8] B. C. Tripathy, B. Chandra Hazarika and B. Choudhary, "Lacunary Iconvergent sequences", Kyungpook Mathematical Journal, vol. 52, no. 4, pp. 473-482, 2012.

[9] B. C. Tripathy and Mausami Sen, "On fuzzy I-convergent difference sequence spaces", Journal of intelligent \& fuzzy systems, vol. 25, no. 3, pp. 643-647, 2013. 
[10] E. Savaş and M. Mursaleen, "On statistical convergent double sequences of fuzzy numbers", Information Sciences, vol. 162, no. 3-4, pp. 183-192, 2004.

[11] H. Fast, "Surla convergence statistique", Colloquium mathematicae, vol. 2, no. 3-4, pp. 241-244, 1951.

[12] I. J. Schoenberg, "The intregrability of certain functions and related summability methods", The American mathematical monthly, vol. 66, no. 5, pp. 361-375, 1959.

[13] K. T. Atanassov, "Intuitionistic fuzzy sets", Fuzzy Sets and Systems, vol. 20, pp. 87-96, 1986.

[14] L. A. Zadeh, "Fuzzy sets", Information and Control, vol. 8, no. 3, pp. 338-353, 1965.

[15] M. Mursaleen and Q. M. D. Lohni, "Intuitionistic fuzzy 2-normed space and some related concepts", Chaos, Solitons \& Fractals, vol. 42, no. 1, pp. 224-234, 2009.

[16] M. Mursaleen and O. H. H. Edely, "Statistical convergence of double sequences", Journal of Mathematical Analysis and Applications, vol. 288, no. 1, pp. 223-231, 2003.

[17] P. Das, P. Kostyrko, W. Wilczynski, and P. Malik, "I and I*- convergence of double sequences", Mathematica Slovaca, vol. 58, no. 5, pp. 605-620, 2008.

[18] P. Kostyrko, T. Salat, and W. Wilczynski, "I-convergence”, Real analysis exchange, vol. 26, no. 2, pp. 669-686, 2000.

[19] R. Giles, "A computer program for fuzzy reasoning", Fuzzy sets and systems, vol. 4, no. 3, pp. 221-234, 1980.

[20] R. Saddati and J. H. Park, "On the intuitionistic fuzzy topological spaces”, Chaos, Solitons \& Fractals, vol. 27, no. 2, pp. 331-4, 2006.

[21] T. Salat, B. C. Tripathy, and M. Ziman, "On some properties of I convergence", Tatra Mountain Mathematical Publications, vol. 28, no. 2, pp. 274-286, 2004.

[22] V. A. Khan, K. Ebadullah, and Yasmeen, "On Zweier I-convergent sequence spaces", Proyecciones (Antofagasta), vol. 33, no. 3, pp. 259-276, 2014. 
[23] V. A. Khan and K. Ebadullah, "Intuitionistic fuzzy Zweier I-convergent sequence spaces", Functional Analysis: Theory, methods and applications, vol. 1, pp. 1-7, 2015.

[24] V. A. Khan and Yasmeen, "Intuitionistic Fuzzy Zweier I-convergent Double Sequence Spaces", New Trends in Mathematical Sciences, vol. 4, no. 2, pp. 240-247, 2016.

[25] V. A. Khan, Yasmeen, "Intuitionistic fuzzy Zweier I-convergent double sequence spaces defined by modulus function", Cogent Mathematics \& Statistics, vol. 3, no. 1, 2016.

\section{Vakeel A. Khan}

Department of Mathematics, Aligarh Muslim University,

Aligarh-202002,

India

e-mail: vakhanmaths@gmail.com

Corresponding author

Mohd. Imran Idrisi

Department of Mathematics, Aligarh Muslim University,

Aligarh-202002,

India

e-mail: mhdimranidrisi@gmail.com

and

\section{Umme Tuba}

Department of Mathematics,

Aligarh Muslim University,

Aligarh-202002,

India

e-mail: ummetuba01@gmail.com 